\title{
An Approach in the Software Testing Environment using Artificial Bee Colony (ABC) Optimization
}

\author{
Tajinder Singh \\ Dept. I.T \\ GIMET, Amritsar.
}

\author{
Mandeep Kaur Sandhu \\ Dept. I.T \\ GIMET, Amritsar.
}

\begin{abstract}
So many techniques are used in the software testing environment and in this paper we survey the $\mathrm{ABC}$ algorithmic approach and its advantages over the GA (Genetic Algorithms). Artificial bee colony (ABC) algorithm is one of the most recently introduced swarm-based algorithms. These optimization approaches helps in memorization and also support the global optima. This algorithm is based on colony size and the maximum number of cycles per number. In this algorithm we know that it is a optimization tool which provides the population based search procedure which is known as Food Sources and these food sources are searched by Employee Bees $\mathrm{ABC}$ as an optimization tool which provides a population-based search procedure in which individuals food positions are modified by different bees with time.
\end{abstract}

Key words-- ABC (Artificial Bee Colony), SUT (Software under test), Software Testing, GA (Genetic Algorithm).

\section{INTRODUCTION}

As we know that Software testing method naturally takes the $50 \%$ of the total cost which is involved in the Software development process[1]. This percentage can be increased if the software is critical [2] and if it takes more time for testing process. So the testing process should be simple and easy to use which consumes less time and efforts for testing process and also gain consumers' confidence in the software. Because testing a software is a costly and time consuming process and the tools present in the market might not be applicable for all the testing conditions and environments because they are useful not at all conditions. So vast interests emerged in swarm intelligence. In the swarm intelligence optimization mostly used methods are ant colony optimization, bacterial foraging optimization algorithms, and bee colony algorithms. Artificial bee colony (ABC) algorithm is one of the most recently introduced swarm-based algorithms. In the $A B C$ algorithm bees duty is to looking for flowers and try to find the best place of flowers. This process is distributed in a asynchronous process means in this process the bees exchange their information with each other about the food sources after dancing on the searched sources of food. In ABC software test optimization we have different types of bees which are called as
A. $\quad$ Employed Bee
B. Onlooker Bee and
C. Scouts Bee

These the kind of bees perform in a different manner and the working of these bee is describes as:

\section{A. Employed Bee}

The purpose of the Employe Bee is to goes to their food sources and then come back to their hive and dances on this area.

\section{B. Scout Bee}

The employee bee becomes scout whose food source has been abandoned. Sometimes scouts bee fly and wish to adopt the food source randomly without using experience then if the amount of nectar at the new food source is higher than the previous food source then the scout bee remember and stores the new position of food and forget the previous one from their memory.

\section{Onlookers}

The onlookers choose the food source depending on the dance of employed bees.

\section{Working of ABC Software Test Optimization:}

In the $\mathrm{ABC}$ approach the following steps are followed for the optimization:

(i) In the first step of test optimization the food sources are produced for all the employed Bees.

Repeat

(ii) After the generation of food sources the employee bee goes to the food source and it checks the amount of nectar present at the food source. It evaluates the nectar amount with the neighbor food source and then come back to their hive and dances in the hive.

(iii) Onlooker watches the dance of employed bees and chooses the food source according to the dance of employed bee. When onlooker goes to that food source and then it evaluates its nectar amount with their neighbor nectar amount.

(iv) Then the vacant food sources are determined and they are replaced with the new food sources and these new food sources are discovered but Scout Bee.

(v) The food source having best food depending upon the nectar amount is to be registered

(vi) Until (Requirements are meet) 


\section{Framework of ABC Test Optimization}

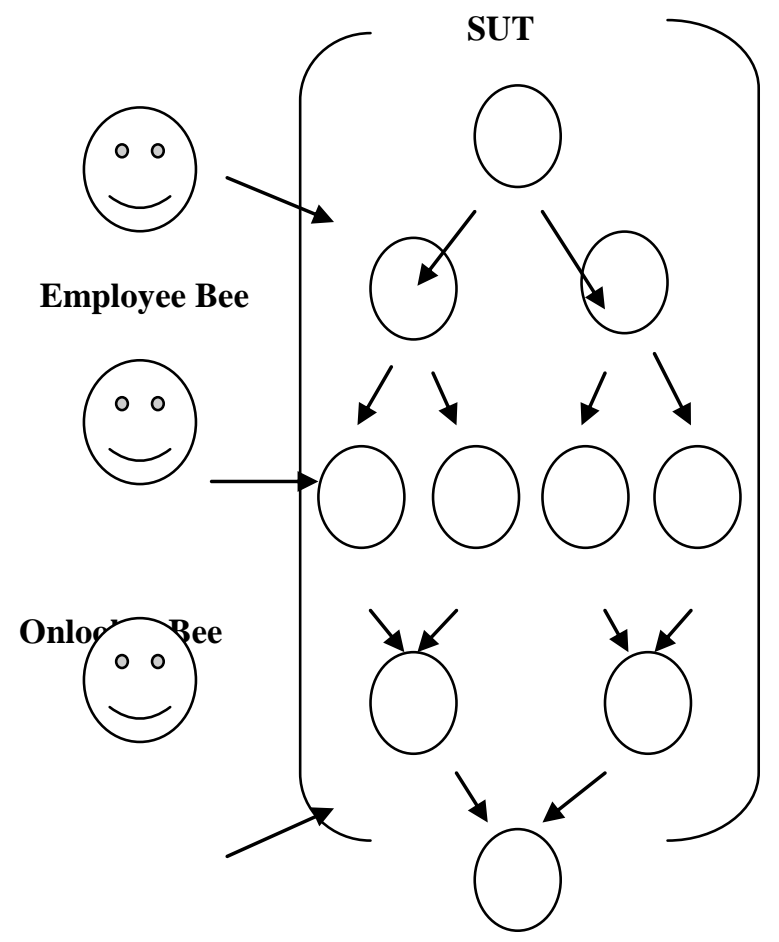

Scout Bee

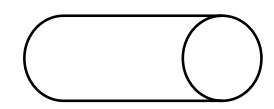

Warehouse of

Test Suite

Optimized

Fig 1: 'Framework of ABC Test Optimization'

$\mathrm{ABC}$ algorithms is defined by Dervis Karaboga in 2005, which is based on the intelligent behavior of honey bees. This algorithm is based on colony size and the maximum number of cycles per number[3]. In this algorithm we know that it is a optimization tool which provides the population based search procedure which is known as Food Sources and these food sources are searched by Employee Bees $A B C$ as an optimization tool which provides a population-based search procedure in which individuals food positions are modified by different bees with time. These bees search the new food source having higher nectar amount[3] i.e. finally with the highest nectar amount[3].

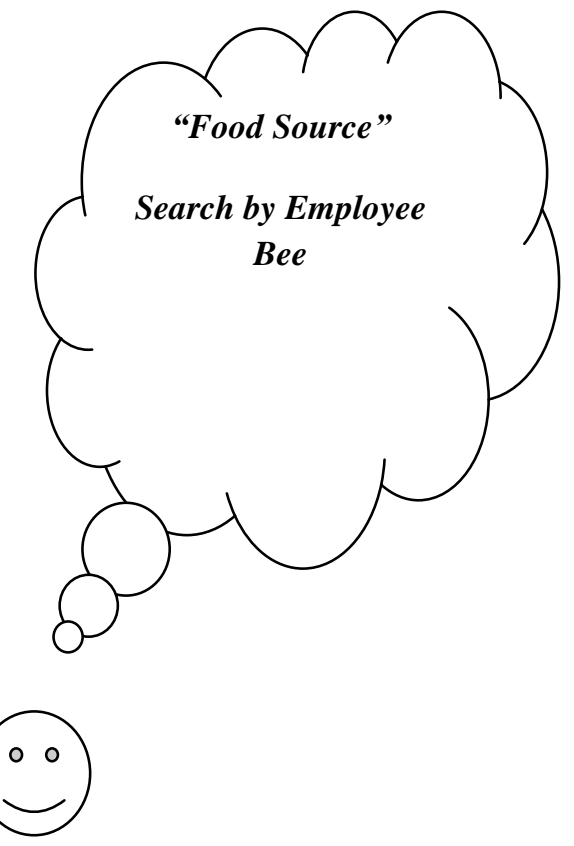

\section{Employee Bee}

Fig 2: 'Food source searched by Employee Bees'

This Diagram represents the food source or dancing area searched by the employee bees. After searching the food source the employee bee will go back to their hive and will start dancing. Depending upon the dance the onlookers' will choose the food source. The employee bee becomes scout whose food source has been abandoned. Now the onlookers' will compares the value of nectar with the neighbor food source is the new food source have higher nectar amount[3] then it will memorize the new food source and forgot about the prior food source. The sources which are abandoned replaced by new food sources one by one by the scouts.

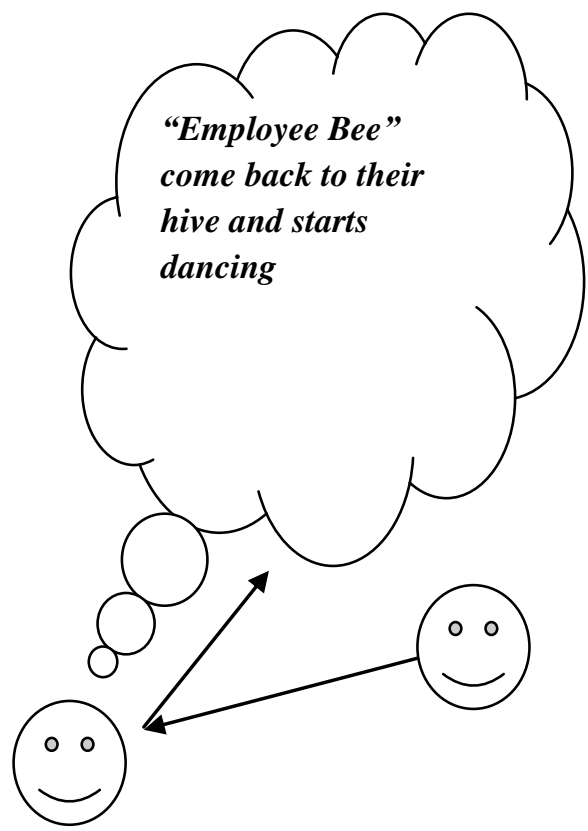

"Onlooker" watching the dance of Employee Bee

Fig 3: 'Onlooker's Process' 


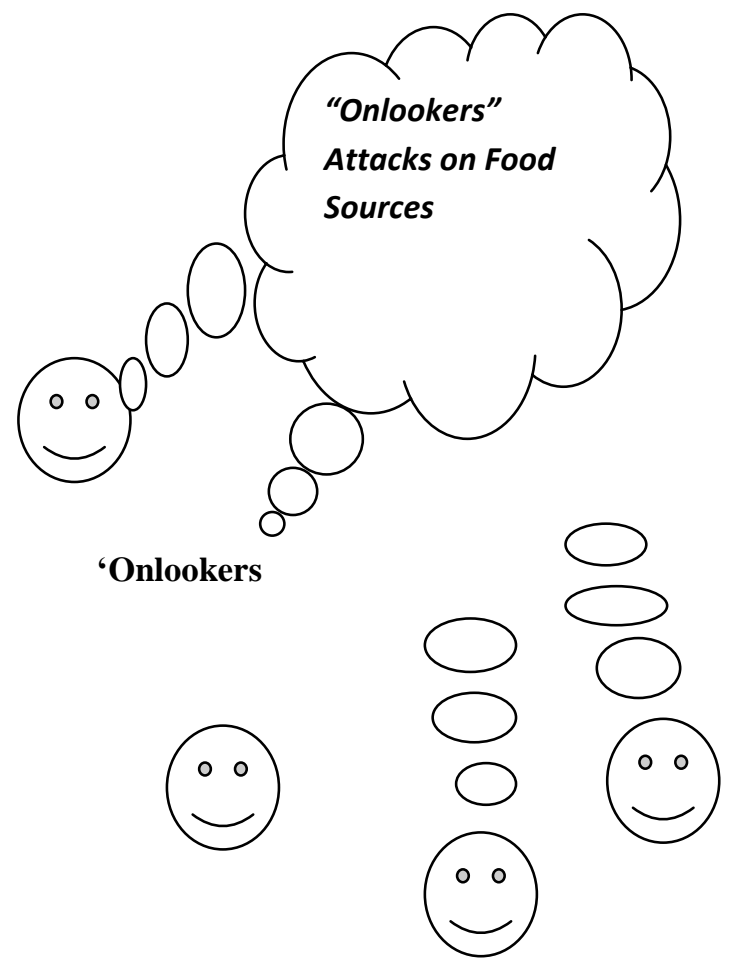

"Onlookers"

Fig 4: 'Attack of Onlooker's on Food Source'

Now when the Onlookers will attack on the food source then that particular food source will become the abandoned and it will be replaced by the new food source by the Scout Bee.

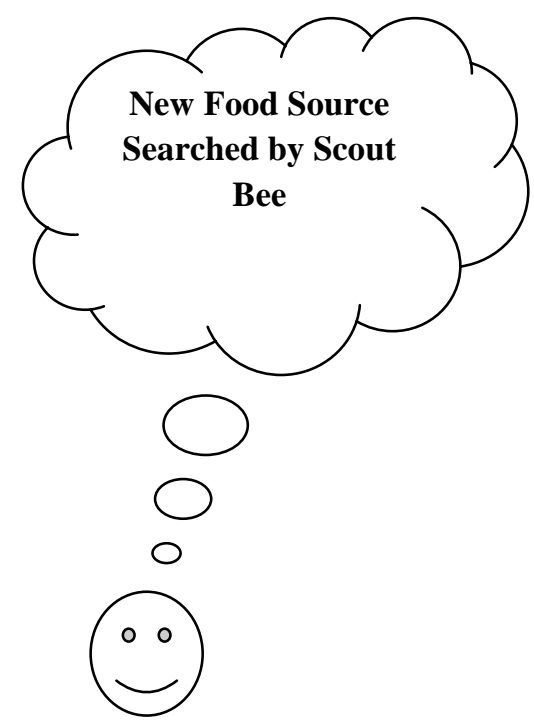

Fig 5: ' New Food Source'

\section{Why ABC Approach for Testing Optimization:}

As our literature describes the drawbacks of the existing systems and in the knowledge based system, so we have to follow a new technique and to focus on the new technique without the problem of local optima. So the Study of ABC Optimization approach have solve out this problem in the
SUT (Software under testing)[4]. So for this advantage of ABC Optimization we can focus on the ABC Approach Which is a NP - hard problem.

\section{Conclusion}

In this paper we describe the behavior and application of $\mathrm{ABC}$ Optimization in the Software test optimization approach. We also describe the different steps of ABC Optimization and our paper describes the framework of software under test (SUT). W e can say that $A B C$ is the a best approach for the software testing which is efficient take less time as compare to the existing GA (Genetic Algorithm) and easy to implement. Because GA have some problems which can be solved by $\mathrm{ABC}$ optimization because GA include no memorization, non linear optimization, risk of suboptimal solution and delayed convergence. GA does not support global optimal solution even when it may be reached. But, ABC model based test suite optimization generates near global optimal results and it converges within less number of test runs. So we can say that $\mathrm{ABC}$ is the best approach in the testing environment of software's.

\section{References}

[1]. D. Jeya Mala, V. Mohan, 2009. ABC Tester - Artificial Bee Colony Based Software Test Suit Optimization Approach, in International .Journal of Software Engineering, IJSE Vol.2 No.2.

[2]. D. Karaboga, B. Basturk, 2007. On The Performance Of Artificial Bee Colony (ABC) Algorithm, Applied Soft Computing, Volume 8, Issue 1, January 2008, pp. 687-697.

[3]. D. Karaboga, 2005. An idea based on beeswarm for numerical optimization, Tech. Rep. TR-06, Erciyes University Engineering Faculty, Computer Engineering Department.

[4]. F. Gao, J.-J. Lee, Z. Li, H. Tong, and X. L“u, 2009. Parameter estimation for chaotic system with initial random noises by particle swarm optimization, Chaos, Solitons \& Fractals, vol. 42, no. 2, pp. 1286-1291.

[5] Hélène Waeselynck, Pascale Thévenod-Fosse, Olfa Abdellatif-Kaddour, 2007. Simulated annealing applied to test generation: landscape characterization and stopping criteria, Empirical Software Engineering, Vol.12, No.1, pp.35-63.

[6]. Michael Grottke, Kishor S. Trivedi, 2007.Fighting Bugs: Remove, Retry, Replicate, and Rejuvenate, IEEE Computer Vol.40 No.2, pp. 107-109.

[7]. Mohammad Fathian, Babak Amiri and Ali Maroosi, 2007. Application of honey-bee Mating optimization algorithm on clustering, Applie Mathematics and Computation, Volume 190, Issue 2, pp. 1502-1513.

[8]. Rudolf Ramler, Klaus Wolfmaier, 2006.Economic perspectives in automation balancing automated and manual testing with opportunity cost, Proceedings of the international workshop on Automation of Software Test, pp.15-23.

[9]. Tracey, N., Clark, N., .Mander K., and McDermid, N., 2002. A Search Based Automated Test Data Generation Framework for Safety Critical Systems, in Systems Engineering for Business Process Change (New Directions), Henderson P., Editor, Springer Verlag.

[10]. Y. Chen, X. Chen, and S. Gu, 2007. Lag synchronization of structurally nonequivalent Chaotic systems with time delays, Nonlinear Analysis: Theory, Methods \& Applications, vol. 66, no. 9, pp. 1929-1937. 